\title{
Ectopic Intrathoracic Kidney Along with Right Anterior Diaphragmatic Hernia
}

\author{
Fateme Hosseinabadi ${ }^{1}$, Ali Hekmatnia ${ }^{2}$, Farzaneh Hekmatnia ${ }^{3}$ and Aidin Lotfiazar ${ }^{4,}$ \\ ${ }^{1}$ Department of Radiology, School of Medicine, Zahedan University of Medical Sciences, Zahedan, Iran \\ ${ }^{2}$ Department of Radiology, Alzahra Hospital, Isfahan University of Medical Sciences, Isfahan, Iran \\ ${ }^{3}$ Department of Medicine, Southend University Hospital, Southend-on-Sea, UK \\ ${ }^{4}$ School of Medicine, Zahedan University of Medical Sciences, Zahedan, Iran \\ "Corresponding author: Resident of Radiology, School of Medicine, Zahedan University of Medical Sciences, Zahedan, Iran. Email: aidinlotfiazar@gmail.com
}

Received 2019 January 11; Revised 2019 January 26; Accepted 2019 February 16.

\section{Dear Editor,}

Intra-thoracic kidney (ITK) is a very rare congenital disease which is considered as an uncommon type of renal ectopia (1). ITK accounts for $0.1 \%$ of the total renal ectopia cases and it is twice as common in men as women. Additionally, ITK in the left hemi thorax is twice as common as on the right (2). Although ITK can be asymptomatic in the most of the cases, it is important to distinguish it from other significant conditions, such as pulmonary masses etc. (3). In this article we report a 5 years old female patient with right sided ITK which presented with chronic respiratory symptoms.

A 5-year-old female was brought to the general surgery clinic with chronic respiratory symptoms including occasional non-productive coughs since birth. The patient was referred to the radiology department and chest X-ray was carried out. There was no history of fever, trauma, surgery or underlying disease (such as renal dysfunction, respiratory distress or gastrointestinal diseases). On the chest Xray, there was an ill-defined opacity in the lower zone of the right hemi thorax (Figure 1). In the abdominal sonography, there was no right kidney in the anatomical position and pelvis. Also, there was a suspicious view of the diaphragmatic defect in the anterior part of the diaphragmatic muscle in the right hemi-thorax.

Therefore, the patient underwent magnetic resonance urography (MRU), as the best radiation-free method, to confirm the diagnosis of intrathoracic renal ectopia. On MRU, there was a right ITK and intrathoracic intestinal herniation (Figure 2).

Renal development during the embryonic period divides into three stages: pronephros, mesonephros and metanephros (4). During the pronephros, which takes up to four weeks of the embryonic period, primary nonfunctional kidneys appear. Thereafter, the mesonephros period begins with functional kidneys developing in the embryo which takes up to nine weeks of embryonic period. These kidneys appear around the vertebral column in the form of two ductal structures. After this stage, metanephros begins with the formation of metanephrogenic blastema (which develops into permanent kidneys) and ureteric bud (which develops into collecting systems) in the pelvis and begin to rise in their pathway in retroperitoneum to renal fossa (4). Any defect along this pathway (includes hypo acceleration, over acceleration and metanephrogenic blastema fusion) can lead to ectopic kidneys including pelvic, horseshoe, crossed or thoracic kidney.

Previous research has suggested some predisposing factors such as maternal vitamin A or folic acid deficiency, radiologic exposure, infectious agents in fetal period, teratogens, 18 trisomy and 21 trisomy for developing renal ectopia (5).

The prevalence of this disease has been reported less than one in 1000 births (5). Most of the ectopic kidneys are located in the pelvis or lower lumbar region. ITK is the rarest type of renal ectopia and has been reported one in 10000 cases of renal ectopia (6).

It can be diagnosed in the prenatal and postnatal period, sonography, X-ray, computed tomography (CT) scan, CT urography, magnetic resonance imaging (MRI) and scintigraphy are diagnostic methods for renal ectopia evaluation; however, ultrasound is still at the most costeffective, non-invasive and reliable method for screening (7).

ITK was first described in 1930 by Campbell (8). The incidence of ITK has been reported 0.5 to $5 \%$ of renal ectopia and occurs in less than five in one million live births $(9,10)$. It is also more common in males and left hemi thorax than females and right hemi thorax. This condition has a strong association with the Buchdalek or Morgani hernia (11). This type of ectopic kidney usually has normal function and its 

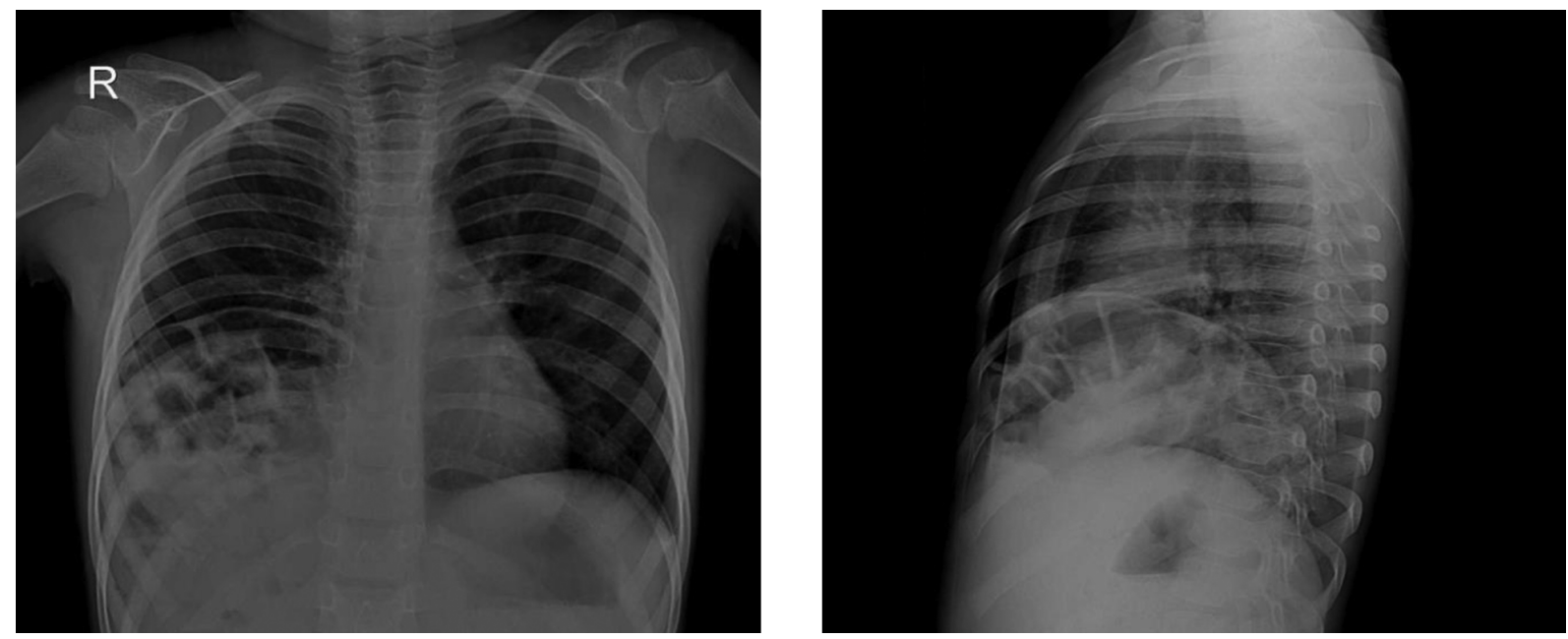

Figure 1. In the anterior posterior and lateral chest X-ray, the right hemi-diaphragm is obscured and elevated. There are multiple loop-like lucencies at the right lower lung zone, suspicious of diaphragmatic hernia, associated with an ill-defined opacity.
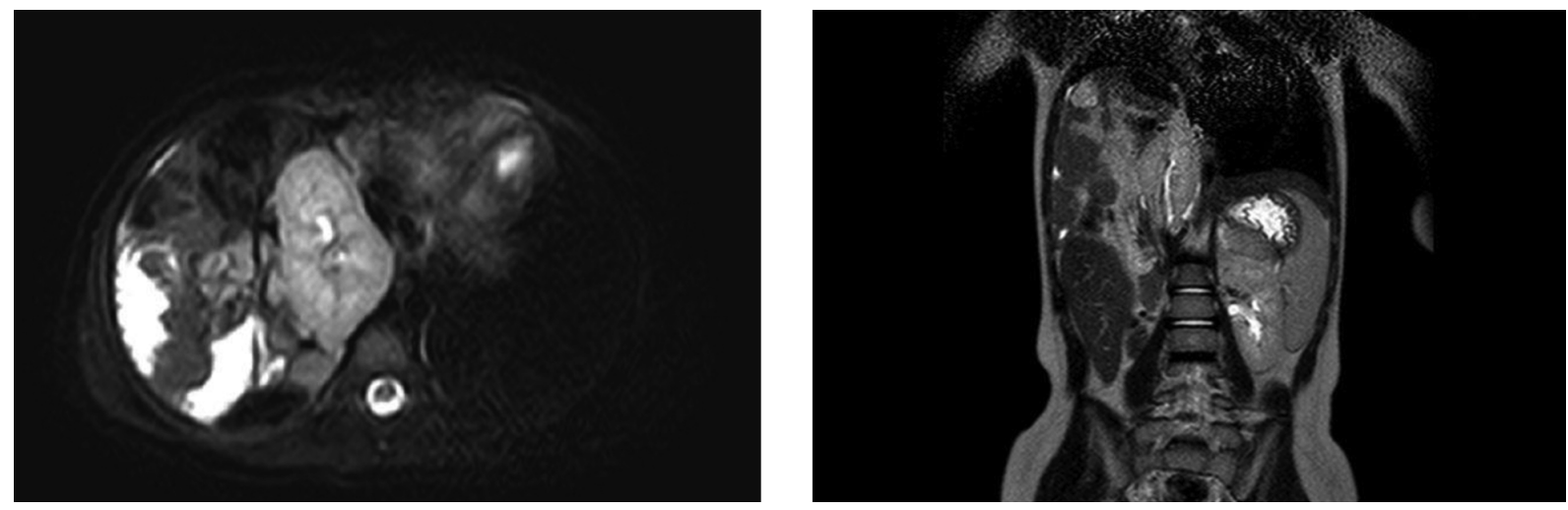

Figure 2. Axial and coronal MRU demonstrate a malrotated right intra-thoracic kidney with a long ureter and high origin of renal vessels. There is also herniation of bowe loops into the posterior mediastinum. The right renal artery originates from the abdominal aorta and the ureters can be seen passing through the diaphragmatic hiatus.

blood supply can be maintained from the abdominal aorta or the thoracic aorta (12).

When there is a combination of ITK and bowel herniation, the surgical nephropexy and diaphragmatic repair are considered appropriate, but in the case of pure ITK, observation has been recommended (12).

In terms of radiological studies, abnormal shape, intrathoracic origin of vessels, longer ureter, abnormal rotation and medial deviation of lower pole of the kidney have been observed (13).

ITK is classified into four groups based on its features: (1) Pure ITK with normal function, (2) ITK with diaphragmatic eventration, (3) ITK with diaphragmatic hernia which could be congenital (Type A) or acquired (Type B), and (4) Post traumatic diaphragmatic rupture and in- trathoracic renal herniation (14).

In this case, there was an uncommon presentation of the ITK. As we discussed above, ITK is more common in males and in left hemi-thorax while in our case it was in a female and the right hemi-thorax. One of the reasons that can be considered for the higher incidence of ITK in the left hemi-thorax can be the presence of the liver on the right which acts as a mechanical barrier against the hyper acceleration of the kidney in embryonic period. An alternative explanation, can be earlier closing of the right hemi thorax diaphragm than the left (15).

Moreover, in this case, there was an anterior diaphragmatic hernia of the intestinal loops. There was a few reports of this coincidence of hernia and ITK but none of the studies reported the incidence of this type of ITK(16). 
In conclusion, identifying the ITK subtype, based on radiological studies, is essential for treatment and management planning.

\section{Acknowledgments}

Authors express their thanks to doctor Sharareh Sanei Sistani for her kindly guides to write this article.

\section{Footnotes}

Authors' Contribution: Study concept and design, diagnosis, critical revision of the manuscript for important intellectual content and study supervision: Ali Hekmatnia and Fateme Hosseinabadi; drafting of manuscript and acquisition of data: Farzaneh Hekmatnia and Aidin Lotfiazar; English and scientific content edition: Farzaneh Hekmatnia; corresponding author: Aidin Lotfiazar. All of the authors read and approved the letter.

Conflict of Interests: The authors declare that there are no conflicts of interest in this study.

Ethical Consideration: This study follows the ethical standards of declaration of Helsinki.

Funding/Support: There was no organization for funding in this study.

Patient Consent: The written patient consent was made for this research.

\section{References}

1. Szmigielska A, Ksiezopolska A, Roszkowska-Blaim M, Brzewski M, Krzemien G. Rare renal ectopia in children - intrathoracic ectopic kidney. Dev Period Med. 2015;19(2):186-8. [PubMed: 26384121].

2. Sarac M, Bakal U, Tartar T, Canpolat S, Kara A, Kazez A. Bochdalek hernia and intrathoracic ectopic kidney: Presentation of two case reports and review of the literature. Niger J Clin Pract. 2018;21(5):681-6. doi:10.4103/njcp.njcp_217_17. [PubMed: 29735873].
3. Sharma P, Keenan RJ, Sexton WJ. Mass in solitary intrathoracic kidney within bochdalek hernia. Urology. 2016;97:e15-6. doi: 10.1016/j.urology.2016.08.017. [PubMed: 27554626].

4. Mikuz G. [Ectopias of the kidney, urinary tract organs, and male genitalia. German version]. Pathologe. 2018;39(5):415-23. German. doi: 10.1007/s00292-018-0474-2. [PubMed:30135974].

5. Magak P, King $\mathrm{CH}$, Ireri E, Kadzo H, Ouma JH, Muchiri EM. High prevalence of ectopic kidney in Coast province, Kenya. Trop Med Int Health. 2004;9(5):595-600. doi: 10.1111/j.1365-3156.2004.01228.x. [PubMed: 15117304]

6. Eid S, Iwanaga J, Loukas M, Oskouian RJ, Tubbs RS. Pelvic kidney: A review of the literature. Cureus. 2018. doi:10.7759/cureus.2775.

7. Chang PL, Mrazek-Pugh B, Blumenfeld YJ. Prenatal diagnosis of crossfused renal ectopia: does color Doppler and 3-dimensional sonography help? JUltrasound Med. 2011;30(4):578-80. [PubMed: 21460160].

8. Campbell MF. Renal ectopy. J Urol. 1930;24(2):187-98. doi: 10.1016/s0022-5347(17)72892-3.

9. Chong SL, Chao SM. An unusual cause of mediastinal mass - A case report and literature review of intrathoracic kidney. Proc Singapore Healthc. 2012;21(2):144-50. doi: 10.1177/201010581202100209.

10. Al-Saqladi AW, Akares SA. Intrathoracic kidney in a child with literature review. Saudi J Kidney Dis Transpl. 2015;26(2):349-54. [PubMed: 25758888].

11. Peterson JLH, Riggs H, Nedungadi S, Lansdale N, Kamupira S. Intrathoracic renal ectopia with congenital diaphragmatic hernia. Arch Dis Child Fetal Neonatal Ed. 2019;104(3):F320. doi: 10.1136/archdischild2018-316195. [PubMed: 30636694].

12. Murphy JJ, Altit G, Zerhouni S. The intrathoracic kidney: should we fix it? J Pediatr Surg. 2012;47(5):970-3. doi: 10.1016/j.jpedsurg.2012.01.056. [PubMed: 22595583].

13. Panda B, Rosenberg V, Cornfeld D, Stiller R. Prenatal diagnosis of ectopic intrathoracic kidney in a fetus with a left diaphragmatic hernia. J Clin Ultrasound. 2009;37(1):47-9. doi: 10.1002/jcu.20503. [PubMed: 18615674].

14. Pfister-Goedeke L, Brunier E. [Intrathoracic kidney in childhood with special reference to secondary renal transport in Bochdalek's hernia]. Helv Paediatr Acta.1979;34(4):345-57. German. [PubMed: 521300].

15. Maduekwe E, Weinberg B, Samaan B, Sheferaw E, Suntharos P. Right thoracic ectopic kidney in a child with breathing difficultiessonographic diagnosis. J Clin Ultrasound. 2011;39(2):108-10. doi: 10.1002/jcu.20716. [PubMed: 20564312].

16. Lundy SD, Gill BC. Herniated Kidney. Urology. 2019;123:e7-8. doi: 10.1016/j.urology.2018.09.026. [PubMed:30308262]. 